\title{
articles
}

\section{Longer duration of breastfeeding associated with reduced risk of developing schizophrenia}

M S Hartog, MB ChB, MMed (Psych)

P P Oosthuizen, MB ChB, MMed (Psych), FCPsych (SA), Phd

R A Emsley, MB ChB, MMed (Psych), FCPsych (SA), MD

Department of Psychiatry, Stellenbosch University, W Cape

Background. Some studies have suggested that breastfeeding may be protective against the development of schizophrenia. Such a theory supports the neurodevelopmental hypothesis of schizophrenia.

Objective. To determine whether there were differences in breastfeeding patterns between patients with schizophrenia and controls.

Method. Fiffy subjects with schizophrenia and 50 age- and gender-matched controls were included in the study. All subjects and their mothers were interviewed using a structured questionnaire to determine the extent of breastfeeding in infancy and age of onset of psychosis.

Results. There was a significant difference in the duration of breastfeeding in patients versus controls $(p<0.05)$.

Conclusion. Breastfeeding seems to be protective against the development of schizophrenia. This supports the neurodevelopmental hypothesis of this disorder and may be indicative of the role of essential fatty acids in normal brain development and the prevention of schizophrenia.

Schizophrenia has a lifetime prevalence of approximately $1 \%$, although this may vary between locations. It is a serious disease with far-reaching consequences, often with devastating effects on the lives of patients and their families. It is also a major contributor to health care expenditure, to such an extent that the cost of caring for these patients accounts for $2.5 \%$ of all health care expenditure in the USA.

In the last few decades research into schizophrenia has been driven mainly by neurotransmitter hypotheses, the basic presumption being that the disorder is caused by dysregulation in synthesis, release, re-uptake and receptor response /which includes alterations in gene expression) to dopamine, noradrenalin, acetylcholine or excitatory amino acids. ${ }^{2}$ The main support for this hypothesis comes from the efficacy and pharmacological profiles of the current antipsychotic medications. These medications do not, however, treat all the symptom complexes of the disorder, and other mechanisms of disease have received more attention recently. One of the most widely supported theories on the pathophysiology of schizophrenia is the neurodevelopmental hypothesis. In its most simplified form, this theory proposes that interaction between genetic and environmental factors influences the way in which neurons originate, migrate, are differentiated, culled by apoptosis and remodelled. Abnormalities in any of these processes may lead to abnormal brain function, which may in turn manifest as the syndrome of schizophrenia. ${ }^{2}$

Environmental factors that may play a role include those associated with prenatal development (maternal malnutrition, paternal age, intra-uterine infections), early postnatal factors (premature birth, low birth weight, obstetric complications), minority status, substance abuse, traumatic life events and residence in an urban area. ${ }^{3.8}$ Possible correlation between breasffeeding and schizophrenia is another putative environmental factor. ${ }^{9}$ Several studies have suggested that breasffeeding may be a protective factor that may delay or even prevent the onset of schizophrenia. ${ }^{9 \cdot 12}$

\section{Objectives}

The study aimed to determine whether there were any differences in the breastfeeding practices of mothers of patients with schizophrenia compared with non-psychiatric age- and gendermatched controls

\section{Methods}

The study was approved by the Ethics Committee of Stellenbosch University. All subjects signed written, informed consent. Fiffy subjects were recruited from the inpatient wards at Stikland and Tygerberg hospitals in Cape Town.

All subjects met Diagnostic and Statistical Manual for Mental Disorders (DSM-IV) criteria for a diagnosis of schizophrenia.

The following patients were excluded: (i) those with unclear diagnosis, specifically with co-morbid substance abuse; (ii) patients with neurological disorders; (iii) patients known to be 
HIV-positive; (iv) patients with any other known medical condition; and $(v)$ mentally retarded patients and patients with birth defects.

The controls were recruited from the ophthalmology, gynaecology and paediatric outpatient clinics at Tygerberg Hospital, Cape Town.

Controls were individually age-and gender-matched with patients. All controls were recruited from within the state health system and were from the same socio-economic group as the patients. They lived in the same catchment area as the Stikland and Tygerberg hospital psychiatric unit patients. Mothers of the controls completed a questionnaire on the physical and mental health of their offspring (controls) to exclude the possibility of any current or previous psychiatric or physical illness. Children with mental retardation or developmental disorders were also excluded.

\section{Study design}

The investigator (MSH) contacted the mothers of the 50 subjects. Personal interviews were conducted either telephonically or face to face. After obtaining informed consent from both the subjects and their mothers, the following information was sought: (i) age and gender of the patient; (ii) whether the patient was breastfed as an infant - breastfeeding was defined as having been put to the breast at least once; (iii) if the subject was breastfed, the duration of breastfeeding; and (iv) age of onset of schizophrenia.

Controls were recruited from within Tygerberg Hospital. After verbal and written consent had been given, the mothers of controls were asked: (i) the age and gender of controls; (ii) whether the control patient was breastfed as an infant; (iii) if breastfed, the duration of breastfeeding; (iv) whether the control patient suffered from any psychiatric disease; and $(v)$ whether the control patient suffered from any other disease that might affect outcome.

Results are expressed as means \pm standard deviation (SD). Student's ttests were used to compare the groups, and a significance level of 0.05 was used throughout.

\section{Results}

The investigator was able to contact and interview the mothers of all the subjects and controls. The patient group comprised 12 females and 38 males aged $21.7 \pm 8.6$ years, and the control group 11 females and 39 males aged $21.7 \pm 8.6$ years Although not quite reaching statistical significance, numerically fewer patients than controls were breastfed ( $p=0.16$ ) (Fig. 1).

The duration of breastfeeding for the patient group was $8.6 \pm$ 11.31 months and for the control group $14.24 \pm 16.58$ months.

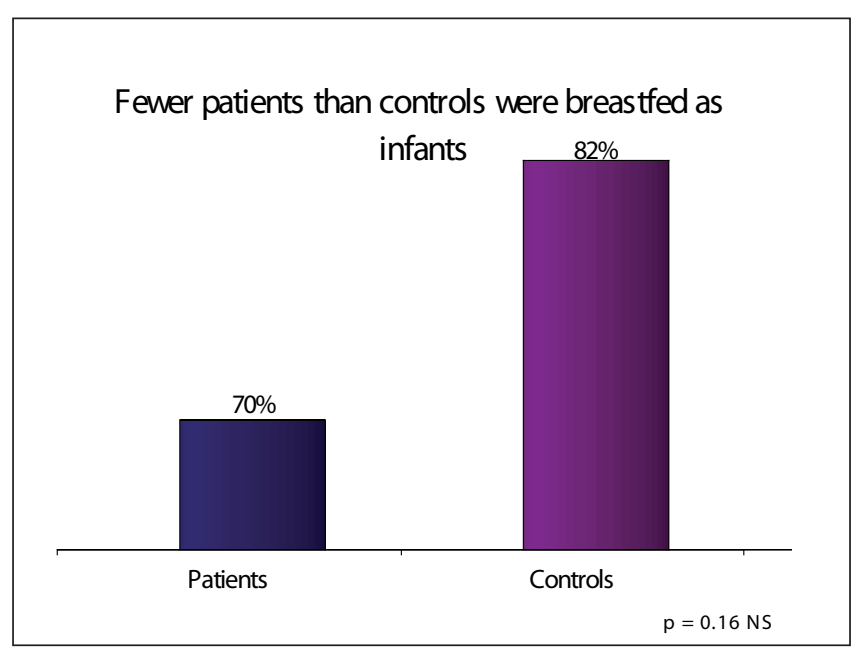

Fig. 1. Percentage of patients and controls breastfed as infants.

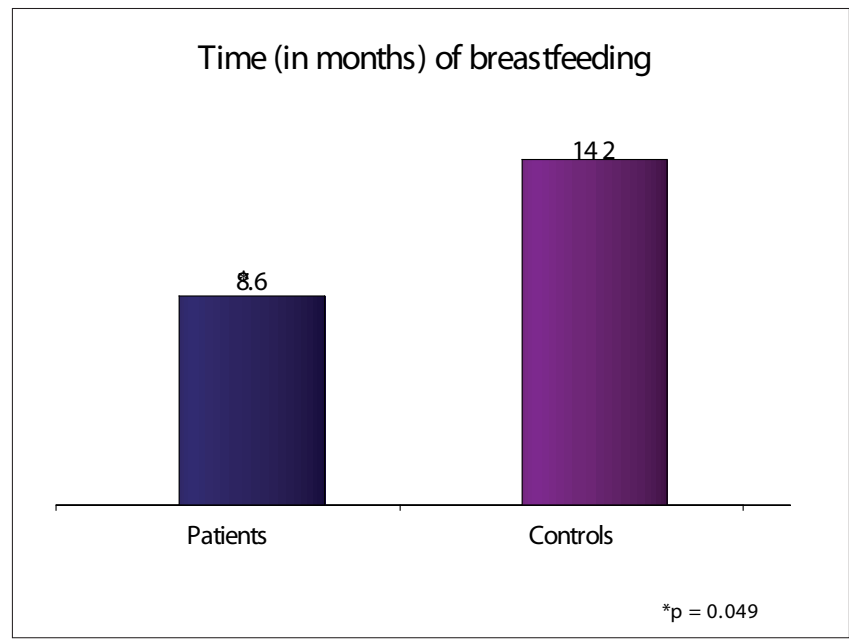

Fig. 2. Duration of breastfeeding (months).

This difference was statistically significant ( $p=0.0497$ ) (Fig. 2).

There was not a significant correlation between the duration of breastfeeding and the age of onset of psychosis.

\section{Discussion}

Our findings seem to support the hypothesis that breastfeeding may offer some protection against the later development of schizophrenia. Although we did not find a statistically significant difference in the number of patients who were breastfed compared with controls, there was a trend toward such a difference.

Only a few published studies have addressed the issue of breastfeeding in schizophrenia. McCreadie ${ }^{9}$ conducted a study in which the mothers of 45 schizophrenic patients completed a questionnaire on breastfeeding. There was a clear trend for a lower rate of breastfeeding among patients compared with 
their unaffected siblings (29\% v. 38\%), although these findings did not reach statistical significance. This study did not include a matched control group, but the figures on breastfeeding were compared with population surveys of breastfeeding in the UK and Scotland. The rate of breastfeeding in the patients was significantly lower than the Scottish survey data. Within the patient group those who had not been breastfed had significantly more schizoid and schizotypal traits and poorer social adjustment at ages $5-11$ and 12 - 16 years. The authors suggested that the absence of breastmilk from the infant diet might be a risk factor for the neurodevelopmental form of schizophrenia. However, it needs to be mentioned that at least four other studies published after the McCreadie ${ }^{9}$ study did not support these findings.

In 1997 Peet et al. ${ }^{2}$ compared 55 patients with DSM IV schizophrenia with 55 controls. In the patient group, 33\% were breastfed versus $43 \%$ in the control group. Forty-four per cent of patients versus $67 \%$ of controls were breastfed for more than 4 weeks.

In 2000 Sasaki et al. ${ }^{10}$ found no correlation between breastfeeding and the later development of schizophrenia. They investigated the feeding patterns of 100 schizophrenic patients, 37 of their siblings and 200 age-matched, healthy controls.

In 2000 Leask et al." found 'no association between breastfeeding and adult psychosis in two national birth cohorts'. Thirty cases of DSM-IIIR-defined schizophrenia were identified from the 1946 cohort and by age $43,24.1 \%$ of cases versus $23.6 \%$ of controls were entirely bottlefed. Forty cases of CATEGO nuclear schizophrenia were identified by age 28 in the 1958 birth cohort. Of these cases $24.1 \%$ versus $31.7 \%$ of controls were entirely breastfed.

In 2003 Amore and colleagues ${ }^{12}$ did a study comparing the breastfeeding history of 113 patients with 140 of their siblings and 113 healthy controls. There were no significant differences between the groups, although a positive correlation was found between duration of breastfeeding and age of onset of illness.

Although the potential mechanisms by which breastmilk may play a protective role are numerous, our findings are compatible with the membrane phospholipid hypothesis of schizophrenia. The latter hypothesis suggests that disturbed phospholipid metabolism is the fundamental cause of schizophrenia. It is postulated that in individuals who develop schizophrenia there is an accelerated rate of loss of unsaturated fatty acids, notably arachidonic acid $(\mathrm{AA})$, docosahexanoic acid (DHA) and eicosapentanoic acid (EPA) ${ }^{2}$
When present to a mild degree, the increased rate of loss will be compensated for by an increased incorporation of phospholipids into the membrane, with no loss of phospholipid composition, but when present to a greater degree, or in association with problems in incorporation, there will be an actual change in membrane composition ${ }^{2}$ which may then manifest later as the syndrome of schizophrenia.

The human brain undergoes a growth spurt during the last trimester of pregnancy and the first few months postnatally. ${ }^{13}$ This requires an abundant supply of essential fatty acids (EFAs). While in utero, the baby gets its supply of EFAs from the mother, but after birth adequate amounts must be supplied in feeds. Human milk contains small but significant amounts of polyunsaturated fatty acids (PUFAs). PUFAs are present in the milk of lactating women throughout lactation. The total percentage of saturated fatty acids progressively increases with time, while the total amount of monounsaturated fatty acids in breastmilk decreases during the first 12 months of lactation. The concentrations of DHA and AA remain stable throughout the nursing period. ${ }^{14}$

It is well established that pre-term and full-term infants fed on formula feeds have lower plasma and erythrocyte levels of DHA and AA than breastfed infants. ${ }^{15,16}$ Several studies ${ }^{17-19}$ have shown a correlation between infant feeding and the development of neuropsychiatric disorders. There is further, growing evidence that functional neurodevelopment is abnormal in infants and children who later develop schizophrenia. PUFAs, particularly AA and $\mathrm{DHA}$, are essential to brain development, and a relative lack of these PUFAs has been associated with cognitive and neurological deficits similar to those seen in bottlefed preterm infants. ${ }^{20}$ Any such predisposition is likely to be exacerbated by a poor dietary supply of PUFAs. ${ }^{20}$

Other mechanisms by which breastfeeding and schizophrenia may interact should clearly also be considered. These would include: (i) the psychological effects of breastfeeding on both mother and infant with effects on bonding and nurturing; ${ }^{21}$ (ii) the possibility that minor neurological abnormalities, so common in patients with schizophrenia, may make it difficult or even impossible for the mother to breastfeed; ${ }^{8}$ and (iii) shared risk factors such as poor mothering skills or adverse home environment which may lead to reduction in the chance of the infant being breastfed as well as having psychological consequences that may be risk factors for schizophrenia. ${ }^{20}$

\section{Conclusion}

Our study reports modest evidence for an association between breastfeeding and schizophrenia. Compared with controls, 
numerically fewer schizophrenic patients were breastfed as infants and the duration of breastfeeding was significantly shorter. Our results support the hypothesis that breastfeeding might be protective against the later development of schizophrenia.

\section{References}

1. Karayiorgou M, Gogos JA. A turning point in schizophrenia genetics. Neuron 1997 19: 967-979.

2. Peet M, Glen I, Horrobin DF. Phospholipid Spectrum Disorder in Psychiatry. Lancashire: Marius Press, 1999: 159-164.

3. McNeil TF, Kaii L. Obstetric factors in the development of schizophrenia: complications in the births of preschizophrenics and in reproduction by schizophrenic parents. In Wynne LC, Cromwell RL, Matthysse S, eds. The Nature of Schizophrenia: New Approaches to Research and Treatment. New York: John Wiley, 1978: 401 1-429

4. McGrath لע, Murray RM. Risk factors for schizophrenia: from conception to birth. In: Hirsch SR, Weinberger DR, eds. Schizophrenia. Oxford: Blackwell Science, 2003: 232-250

5. Watson CG, Kucala T, Tilleskior C, Jacobs L. Schizophrenic birth seasonality in relation to the incidence of infectious diseases and temperature extremes. Arch Gen Psychiatry 1984; $41:$ 85-90.

6. Susser E, Neugebauer R, Hoek HW, et al. Schizophrenia after prenatal famine. Further evidence. Arch Gen Psychiatry 1996; 53(1): 25-31.

7. Wahlberg KE, Wynne LC, Oja H, et al. Gene environment interaction in vulnerability to schizophrenia. Findings from the Finnish adoptive family study of schizophrenia. Am J Psychiatry 1997; 154: 355-362.

8. Kaplan BJ, Sadock VA. Synopsis of Psychiatry. 9th ed. Baltimore: Lippincott \& Wilkins, 2003: 465-466
9. McCreadie R. Breasiffeeding and schizophrenia; preliminary results and hypothesis. $\mathrm{Br}$ J Psychiatry 1997; 173: 334-337

10. Sasaki T, Okazaki Y, Akaho R, et al. Type of feeding during infancy and later development of schizophrenia. Schizophr Res 2000: 42: 79-82

1 1. Leask SJ, Done DJ, Crow TJ, Richards M, Jones PB. No association between breastfeeding and adult psychosis in two national birth cohorts. Br I Psychiatry 2000; 177 218-221.

12. Amore M, Balista $C, M c C r e a d i e ~ R G$, et al. Can breast-feeding protect against schizophrenia? Case control study. Biol Neonate 2003; 83: 97-101.

13. Dobbing J, Sands J. Timing of neuroblast multiplication in the developing human brain. Nature 1970; 226: 639-640.

14. Maragoni F, Agostoni C, Lammardo AM, Giovanni M, Galli C, Riva E. Polyunsaturated fatty acid concentrations in human hind milk are stable throughout 12 months lactation and provide sustained intake to the infant during exclusive breastfeeding; an Italian study. Br J Nutr 2000; 84: 103-109.

15. Boehm G, Borte M, Bohles HJ, Muller H, Kohn G, Moro G.Docosahexaenoic and arachidonic acid content of serum and red blood cell membrane phospholipids of preterm infants fed breast milk, standard formula and formula supplemented with n3 and n-6 long chain polyunsaturated fatty acids. Eur J Pediatr 1996; 155: 410-416.

16. Koletzko B, Dessi T, Demmelmair H. Arachidonic acid supply and metabolism in human infants born at full term. Lipids 1996; $31: 79-83$

17. Menkes $\mid H$. Early feeding of children with learning disorders. Dev Med Child Neurol 1977: 19: 167-175.

18. Tanoue Y, Oda S. Weaning time of children with infantile autism. J Autism Dev Disord 1989; 19: 425-434

19. Pisacane A, Impagliazzo N, Russo $M$, et al. Breastfeeding and multiple sclerosis. BM 1994; 308: 1411-1412.

20. Peet M, Glen I, Horrobin DF. Phospholipid Spectrum Disorder in Psychiatry. Lancashire: Marius Press, 1999: 159-164.

21. Feldman R, Eidelman Al. Direct and indirect effects of breastmilk on the neurobehaviour and cognitive development of premature infants. Dev Psychobiol 2003: 43: 109-119. 